\title{
Reduced Dimension Modeling of Leading Edge Turbulent Interaction Noise
}

\author{
James Gill, ${ }^{*}$ Xin Zhang $^{\dagger}$ and Phillip Joseph ${ }^{\ddagger}$ \\ Faculty of Engineering and the Environment, \\ University of Southampton, Hampshire, SO16 7QF, UK. \\ Thomas Node-Langlois ${ }^{\S}$ \\ Acoustics and Environment - Numerical Methods, \\ Airbus Operations S.A.S, 31060 Toulouse, France.
}

\begin{abstract}
A computational aeroacoustics approach is used to model the effects of real airfoil geometry on leading edge turbulent interaction noise for symmetric airfoils at zero angle of attack. For the first time, one-component (transverse), two-component (transverse and streamwise), and three-component (transverse, streamwise, and spanwise) synthesized turbulent disturbances are modeled instead of single frequency transverse gusts, which previous computational studies of leading edge noise have been confined to. The effects of the inclusion of streamwise and spanwise disturbances on the noise are assessed, and it is shown that accurate noise predictions for symmetric airfoils can be made by modeling only the transverse disturbances, which reduces the computational expense of simulations. Additionally, the two-component turbulent synthesis method is used to model the effects of airfoil thickness on the noise for thicknesses ranging from $2 \%$ to $12 \%$. By using sufficient airfoil thicknesses to show trends, it is found that airfoil thickness will reduce the noise at high frequency, and that the sound power $P$ will reduce linearly with increasing airfoil thickness.
\end{abstract}

Keywords: computational aeroacoustics, leading edge noise, linearized Euler equations, turbulence synthesis

\section{Nomenclature}

$\lambda \quad$ Vortical gust wavelength

$\boldsymbol{k} \quad$ Wave vector

$\boldsymbol{U} \quad$ Freestream velocity

$\sigma(\boldsymbol{k}) \quad$ Random phase term

$\theta \quad$ Observer angle

$c \quad$ Airfoil chord

$K \quad$ Chord-based reduced frequency

$k^{\text {lim }} \quad$ Max. wavenumber limits

$L \quad$ Turbulent integral length scale

$L, M, N$ Fourier summation limits

$\begin{array}{rllr}{[\mathrm{m}]} & M & \text { Mach number } & \\ & P & \text { Sound power } & {\left[\mathrm{Wm}^{-1}\right]} \\ {\left[\mathrm{m}^{-1}\right]} & r_{0} & \text { Observer radius } & {[\mathrm{m}]} \\ {\left[\mathrm{m}^{-1}\right]} & T & \text { Time } & {[\mathrm{s}]} \\ {[\mathrm{rad}]} & t & \text { Maximum airfoil thickness } & {[\mathrm{m}]} \\ {[\mathrm{deg}]} & v & \text { Transverse gust perturbation } & {\left[\mathrm{m} \cdot \mathrm{s}^{-1}\right]} \\ {[\mathrm{m}]} & W(\boldsymbol{k}) & \text { Turbulent velocity spectrum } & {\left[\mathrm{m}^{3 / 2} \cdot \mathrm{s}^{-1}\right]} \\ & & & \\ {\left[\mathrm{m}^{-1}\right]} & \text { Abbreviations } & \\ {[\mathrm{m}]} & \text { CAA } & \text { Computational AeroAcoustics } & \\ & \text { CROR } & \text { Contra-Rotating Open Rotor } & \end{array}$

*EngD student, Engineering Centre of Excellence, J.Gill@soton.ac.uk.

${ }^{\dagger}$ Airbus Professor of Aircraft Engineering, Engineering Centre of Excellence, Associated fellow, AIAA.

$\ddagger$ Professor of Engineering Acoustics, ISVR.

$\S$ Acoustic engineer, Acoustics and Environment Department, Airbus. 
LEE Linearized Euler Equations

PWL Sound Power Level
SNGR Stochastic Noise Generation and Radiation

[dB] SPL Sound Pressure Level

$[\mathrm{dB}]$

\section{Introduction}

$T^{\text {HE }}$ noise produced by the interaction between oncoming vortical disturbances and the leading edge of 1 an airfoil remains a subject of interest in the aeroacoustics community. Turbulent interaction noise is a significant contributor to the noise in turbo-machinery applications, and has also been studied with regards to wind turbine noise. This noise mechanism is also receiving renewed interest because it is a major broadband noise source in contra-rotating open rotor engines (CRORs), which have potential to help meet Flightpath 2050 targets. These targets call for $75 \%, 90 \%$, and $65 \%$ reductions in $\mathrm{CO}_{2}, \mathrm{NO}_{x}$, and noise emissions respectively, against 2000 baselines. ${ }^{1}$ Unfortunately, CRORs are known to produce high levels of tonal and broadband noise. Therefore, studies to understand and to minimize turbulent interaction noise would be beneficial.

Considerable work exists on the subject of predicting turbulent interaction noise with analytical models. Early work by Sears ${ }^{2}$ has been extended to $2 \mathrm{D}$ compressible flow problems by Graham ${ }^{3}$ and Amiet ${ }^{4}$ for example. Amiet ${ }^{4}$ used flat plate theory to predict the noise due to an isolated airfoil interacting with an oncoming turbulent inflow. Amiet's theory still forms the basis of many current industrial prediction models. However, the effect of real airfoil geometry on turbulent interaction noise has not been fully addressed by this theory. In previous studies, a variety of experimental, computational aeroacoustic (CAA), and analytic methods have been used to test the effects on noise when assuming flat plate geometry as opposed to modeling realistic airfoil shapes, but these studies have not yet investigated all aspects. These studies are summarized in Section II.

The current work will investigate modeling assumptions that are frequently used in analytical and computational models of turbulent interaction noise, aiming to show the effects that these assumptions have on noise predictions. By using a CAA code to solve the linearized Euler equations (LEEs), the following will be addressed:

- The effect of symmetric airfoil thickness on the turbulent interaction noise when modeling two-component synthesized frozen turbulent gusts convecting with a steady meanflow.

- The importance of transverse, streamwise, and spanwise disturbance components when modeling turbulent interaction noise.

- The accuracy of turbulent interaction noise predictions made with a reduced dimension modeling approach.

A schematic of a vortical gust interacting with an airfoil is shown in Figure 1.

\section{Previous Work}

Previous experimental, CAA, and analytic studies have tested the effects on noise when assuming flat plate geometry as opposed to modeling a realistic airfoil shape. These have typically concentrated on the effects of airfoil thickness, angle of attack and camber. The effects of airfoil camber and angle of attack are not considered here because they have been shown as small by authors such as Devenport et al. . $^{5}$ The effects of airfoil thickness on the noise will now be summarized. A more detailed discussion of previous studies is given in Ref. ${ }^{6}$

\section{II.A. Experimental Studies}

Paterson and Amiet ${ }^{7}$ experimentally measured the response from an isolated NACA 0012 airfoil due to interactions with nearly isotropic turbulence. By comparing the NACA 0012 noise data with a flat plate analytical model, this was also one of the first studies to note that real airfoil thickness and leading edge geometry can reduce the noise at high frequencies (by $\sim 5 \mathrm{~dB}$ ). However, no trends with varying thickness geometry were explored. The $5 \mathrm{~dB}$ reduction in noise due to the thickness of the NACA 0012 airfoil was measured at a thickness-based reduced frequency $K_{t}=t / \lambda=1$ (where $t$ is the airfoil thickness and $\lambda$ is the vortical gust wavelength). This frequency was suggested by Paterson and Amiet as a measure of when flat 
plate theory breaks down and can no longer be considered accurate. A later study by Olsen and Wagner ${ }^{8}$ considered multiple airfoils and reported a linear trend between the effects on the noise and increasing frequency and airfoil thickness. Devenport et al. ${ }^{5}$ and Hall et al. ${ }^{9}$ also found a reduction in turbulent interaction noise with increasing airfoil thickness. The apparent linear increase in the noise reduction effects with both thickness and frequency was also reported by Roger and Moreau. ${ }^{10}$ They compared measurements from several studies and found that the noise reduction effects collapsed to a single curve when thickness, flow speed, and the turbulent length scale were accounted for.

\section{II.B. Analytical Studies}

Most analytic models for the prediction of leading edge gust interaction noise are restricted to flat plates. However, there have been attempts at extending the theory to include real geometry effects. By using a modified Greens function to account for plate thickness, Gershfeld ${ }^{11}$ showed that radiated sound due to turbulent flow at high frequencies could be reduced by increasing the finite thickness of a flat plate. Glegg and Devenport ${ }^{12}$ showed with a conformal mapping approach that the effect of increasing thickness is to reduce the noise at high frequencies. Moreau et al. ${ }^{13}$ modified existing flat plate analytic theory with semi-empirical corrections, based on observations of thickness effects in experimental studies.

\section{II.C. Computational Studies}

Atassi et al. ${ }^{14}$ numerically investigated the effects of thickness on the noise due to a harmonic gust by using a CAA method. They investigated Joukowski airfoils with thicknesses ranging from $3 \%$ to $12 \%$ of the airfoil chord. This study found that in $M=0.5$ flow at reduced frequencies of $K=c / \lambda \sim 1$ or higher, the effect of thickness was to reduce the noise at downstream observer locations, and increase it at upstream locations. Therefore, the basic shape of the directivity was unaltered, but the resulting pattern was skewed towards the upstream direction when compared with flat plate predictions.

Lockard and Morris ${ }^{15}$ performed a CAA study of noise radiated by NACA 4-series airfoils encountering harmonic vortical gusts in the time domain. They used both inviscid Euler and viscous Navier-Stokes calculations to model vortical gust interactions up to $K \sim 1.2$ in $M=0.5$ flow. Lockard and Morris made similar conclusions to Atassi et al., where airfoil thickness caused an upstream skewing of the directivity pattern such that the noise was reduced at downstream observer locations by a greater amount than at upstream locations. Lockard and Morris gave the realistic airfoil curvature and the realistic meanflow solution as the dominant causes for the change in the noise.

Clair et al. ${ }^{16}$ used CAA methods to model the effects of wavy leading edges on turbulent interaction noise. While Clair et al. did not specifically study the effects of constant span airfoil profiles, which are the focus of this paper, their work is mentioned because the computational method that was used is very similar to the methods employed in the current study.

Gill et al. ${ }^{6}$ used a CAA method to study the effects of airfoil thickness and leading edge radius on the noise from symmetric airfoils encountering harmonic gusts at varying frequency. They found that airfoil thickness can cause a reduction in the noise for reduced frequencies above $K=1$, and identified the primary noise reduction mechanism to be the distortion of the gust wavefront due to the presence of velocity gradients in the leading edge stagnation region.

In comparison to the number of experimental and analytic studies, there are fewer CAA studies which investigate the effects of airfoil geometry on turbulent interaction noise. Furthermore, these studies have been restricted to modeling a small number of discrete frequencies using transverse gusts. The use of single component transverse gusts as a simplified turbulent inflow may affect the sensitivity of leading edge noise to airfoil geometry because this approach is limited to modeling gusts with parallel wavefronts and with no transverse $k_{y}$ wavenumber component. Evers and Peake ${ }^{17}$ have shown with an analytic model to 
predict cascade noise that the leading edge noise due to turbulent flow exhibits a weaker sensitivity to airfoil geometry than to flow containing harmonic disturbances. Although the exact effects of simplifying the input disturbance are not yet known, the use of harmonic disturbances is still regarded as useful in revealing general trends between the leading edge noise and changes in airfoil geometry.

\section{Aims of the Current Work}

The studies described above generally agree that airfoil thickness can cause a reduction in turbulent interaction noise. However, the threshold frequency above which the effects of thickness are seen, remains a topic of debate. Paterson and Amiet ${ }^{7}$ suggested that flat plate theory could be considered inaccurate above about $K_{t}=1$. Hall et al. ${ }^{9}$ found the maximum noise reduction due to airfoil thickness to be around $K_{t}=1$. However, Atassi et al..$^{14}$ and Gill et al. ${ }^{6}$ found reductions in the leading edge noise above $K=1$, which corresponds to $K_{t}=8.3$ for a $12 \%$ thick airfoil. By comparing Paterson and Amiet's measurements with predictions from a CAA method, the current work aims to resolve this discrepancy.

It has been noted that predictions from previous CAA methods are not directly comparable to experimental measurements because CAA methods have so far been restricted to considering single component transverse gusts. CAA methods, such as that by Clair et al. ${ }^{16}$ and Gill et al., ${ }^{6}$ are usually limited to onecomponent transverse gusts in order to reduce the computational cost, and because the transverse component is considered to be the most important for turbulent interaction noise.

Amiet $^{4}$ also highlighted the importance of the transverse disturbances. When analyzing the interaction between a flat plate and oncoming turbulence, Amiet showed that the noise could be modeled by considering only the gusts with zero spanwise wavenumber, provided that the semi-span $d$ was significantly larger than the turbulent lengthscale $L$. A flat plate has no physical presence to interact with streamwise disturbances, so these were not included in Amiet's analysis. Therefore, Amiet simplified the modeling of flat plate turbulent interaction noise to include only the transverse turbulent disturbances.

The current work aims to test the implications of the transverse gust simplification by using a CAA method with a synthesized turbulent inflow to study the turbulent interaction noise for real airfoils. The effects on noise due to airfoil thickness were previously studied by the current authors in Ref. ${ }^{6}$ using a transverse gust method. Parts of this study are repeated here by using a two-component turbulent forcing method. This will determine if the presence of streamwise disturbances will alter the thickness effects on the noise. Additionally, noise predictions will be made using methods which generate a one-, two-, and three-component synthesized turbulent flow. This will allow the relative importance of each disturbance component on the noise to be directly compared.

\section{Synthesizing a Turbulent Inflow}

While stochastic methods do not provide exact solutions of the Navier-Stokes equations, they can provide the main features of turbulence such as realistic length and time scales, and energy distributions, at greatly reduced computational cost. There are two main approaches which have been used by previous authors to synthesize turbulence for CAA studies. These approaches consist of the summation of a series of discrete Fourier modes, or the digital filtering of white noise, in order to obtain the desired result.

\section{IV.A. Fourier Mode Summation}

The generation of synthetic turbulence via the summation of a finite number of Fourier modes was first introduced by Kraichnan. ${ }^{18}$ This method expressed the velocity field as a summation of discrete harmonic frequency disturbances, and generated a $2 \mathrm{D}$ or $3 \mathrm{D}$ velocity field that was divergence-free, statistically stationary, homogeneous, and isotropic. The amplitude of the disturbances was proposed to follow an energy spectrum which Kraichnan prescribed as either a Gaussian spectrum (centered at a given wavenumber) or a single wavenumber component via the use of a Dirac delta function.

Kraichnan's method was later modified by other authors to create the family of stochastic noise generation and radiation (SNGR) methods. Bechara et al. ${ }^{19}$ adapted Kraichnan's method to use the more realistic von Kármán turbulent energy spectrum when setting the amplitude of each frequency disturbance. It was also proposed to obtain time decorrelated results by performing a number of independent realizations of the

turbulent field. Bailly and Juvé ${ }^{20}$ extended the method to include convection effects and time dependence 
via a change of reference frame. Billson et al. ${ }^{21}$ also extended the method to model time-correlation and anisotropy of the disturbances.

Turbulence synthesis via Fourier mode summation has been used in a number of applications by previous authors. When developing their methods, Bailly and Juvé, ${ }^{20}$ and Billson et al. ${ }^{21}$ applied this type of turbulence synthesis to the study of jet noise. More recently, Reboul and Polacsek ${ }^{22}$ used a similar method to model the propagation of broadband noise through, and radiated from, a turbofan bypass duct. Clair et al. ${ }^{16}$ also synthesized an upstream one-component turbulent field (transverse velocity components only) when studying the effect of leading edge serrations on airfoil turbulent interaction noise.

Turbulent synthesis methods that are based on Fourier mode summation are generally less complicated than digital filter methods when generating simple flow fields such as homogeneous, isotropic turbulence. For the present study, they also have the advantage of being conceptually similar to harmonic gust methods. However, Fourier mode methods have difficulties representing more complex features of turbulent flow such as inhomogeneity, and can also be computationally expensive if a large number of modes and random numbers is required.

\section{IV.B. Filtering White Noise}

The synthesis of turbulence via the digital filtering of white noise was proposed by Klein et al. ${ }^{23}$ who showed that the method could accurately reproduce turbulence statistics as well as autocorrelation functions. Other authors such as Klein et al., ${ }^{23}$ and Ewert and Edmunds, ${ }^{24}$ extended the approach to develop what is known as the random particle mesh (RPM) method. Dieste ${ }^{25}$ developed a digital filtering model which considered more realistic energy spectra such as the Liepmann or von Kármán spectra, and applied this to the modeling of broadband fan/OGV interaction noise.

The primary advantage of digital filter methods when compared with Fourier based methods is that they can more easily represent complex turbulent flows. Inhomogeneous, anisotropic, and non-stationary flows can be considered without significant changes to the methods being used. Additionally, continuous frequency content can be modeled by digital filter methods, whereas only discrete frequencies can be considered when using Fourier based methods.

\section{Analytical Method}

The analytic model due to Amiet, ${ }^{4}$ modified to allow for $2 \mathrm{D}$ airfoils, is used for validation of the CAA methods adopted in this paper, and also to provide a baseline solution which makes use of the flat plate geometry assumption.

\section{Computational Method}

The CAA method used in the current work has been described in previous work by the current authors in Ref. ${ }^{6}$ It has been adapted here to allow the synthesis of a turbulent inflow. The method uses SotonCAA, which is a high-order CAA code developed within the University of Southampton, and which has been used in previous aeroacoustic studies such as that by Zhang et al. ${ }^{26}$

The turbulent synthesis method was chosen to use a finite summation of discrete Fourier modes, in a method similar to that used by Bechara et al. ${ }^{19}$ Other authors, such as Reboul and Polacsek, ${ }^{22}$ have also used similar methods for CAA applications. This relatively simple method was chosen instead of a digital filter method because only a simple representation of turbulence was required in the study, and because it is conceptually similar to harmonic gust forcing, which the turbulent gust methods will be compared against. The chosen turbulent synthesis method does not provide a time-decorrelated solution. The disturbance will be periodic about the lowest $k_{x}$ disturbance frequency. Therefore, a number of separate realizations of each simulation must be made, where each realization uses a different set of random numbers $\sigma(\boldsymbol{k})$. Each realization then produces a far-field pressure value $p_{r}\left(r_{0}, \theta, K\right)$, and the final incoherent result is found by averaging across all separate realizations. The averaged result is found from

$$
p\left(r_{0}, \theta, K\right)=\sqrt{\frac{1}{R} \sum_{r=1}^{R}\left|p_{r}\left(r_{0}, \theta, K\right)\right|^{2}}
$$


where $R$ is the total number of realizations, and $r$ is the realization number. As the number of separate realizations increases, the statistical error due to the random phases in each gust mode is reduced. The statistical error was found to reduce proportionally to $1 / \sqrt{R}$, as reported by other authors using similar methods. $R=20$ was used in the current work.

Three turbulence synthesis approaches are used in the current work, in order to produce a one-, two-, and three-component synthesized turbulent disturbance. The implementation of the one-, two-, and threecomponent turbulence synthesis methods will now be described.

\section{VI.A. Three-Component Turbulent Forcing}

Three-component vortical gusts with velocity components in the transverse, streamwise, and spanwise directions, were defined as

$$
\boldsymbol{u}_{\mathbf{3} C}(\boldsymbol{x}, T)=\sum_{l=1}^{L} \sum_{m=-M}^{M} \sum_{n=-N}^{N} W_{3 C}\left(\boldsymbol{k}_{l, m, n}\right) \cos \left(\boldsymbol{k}_{l, m, n}(\boldsymbol{x}-\boldsymbol{U} T)+\sigma\left(\boldsymbol{k}_{l, m, n}\right)\right) \boldsymbol{\zeta}\left(\boldsymbol{k}_{l, m, n}\right),
$$

where $U$ is the freestream velocity, $T$ is time, $l, m, n$ are the summation modes in the streamwise, transverse and spanwise direction respectively, $\boldsymbol{k}_{l, m, n}$ and $\boldsymbol{\zeta}_{l, m, n}$ are the wave vector and direction of each combination of $l, m$, and $n$ modes, $\sigma\left(\boldsymbol{k}_{l, m, n}\right)$ is a randomly generated phase term that is assigned to each mode and is unique to each realization, and $W_{3 C}\left(\boldsymbol{k}_{l, m, n}\right)$ is set to follow the 3D Liepmann spectrum ${ }^{27}$ as

$$
W_{3 C}(\boldsymbol{k})=\sqrt{\Delta k_{y} \Delta k_{z} \frac{2 \overline{w^{2}} L^{3}}{\pi^{2}} \frac{L^{2}\left(k_{x}^{2}+k_{z}^{2}\right)}{\left(1+L^{2} k^{2}\right)^{3}}},
$$

where $k=\sqrt{k_{x}^{2}+k_{y}^{2}+k_{z}^{2}}$. To ensure a divergence free flow, $\boldsymbol{\zeta}$ was defined such that $\boldsymbol{k}_{l, m, n} \cdot \boldsymbol{\zeta}_{l, m, n}=0$. Divergence-free forcing is required so that the input disturbance does not interfere with the acoustic results by creating noise. Unless otherwise stated, simulations in the current work used a turbulent intensity of $\sqrt{\overline{w^{2}}}=0.04 U_{x}$, and a turbulent integral lengthscale of $L=0.07 \mathrm{~m}$.

\section{VI.B. Two-Component Turbulent Forcing}

Two-component vortical gusts with velocity components in the transverse and streamwise directions, were defined as

$$
\boldsymbol{u}_{\mathbf{2}}(\boldsymbol{x}, T)=\sum_{l=1}^{L} \sum_{m=-M}^{M} W_{2 C}\left(\boldsymbol{k}_{l, m}\right) \cos \left(\boldsymbol{k}_{l, m}(\boldsymbol{x}-\boldsymbol{U} T)+\sigma\left(\boldsymbol{k}_{l, m}\right)\right) \boldsymbol{\zeta}\left(\boldsymbol{k}_{l, m}\right) .
$$

In order to obtain $W_{2 C}\left(\boldsymbol{k}_{l, m}\right), W_{3 C}\left(\boldsymbol{k}_{l, m, n}\right)$ would ordinarily be integrated with respect to $k_{z}$ to remove the dependence on spanwise wavenumber. However, According to Amiet's hypothesis, ${ }^{4}$ we can expect only disturbances with $k_{z}=0$ to generate noise. One aim of the current work is to test Amiet's hypothesis for real airfoil geometries. Therefore, $W_{2 C}\left(\boldsymbol{k}_{l, m}\right)$ is defined here to be $W_{2 C}\left(\boldsymbol{k}_{l, m}\right)=W_{3 C}\left(k_{x, l}, k_{y, m}, 0\right)$, or

$$
W_{2 C}\left(k_{x}, k_{y}, 0\right)=\sqrt{\Delta k_{y} \frac{2 \overline{w^{2}} L^{3}}{\pi^{2}} \frac{L^{2} k_{x}^{2}}{\left(1+L^{2}\left(k_{x}^{2}+k_{y}^{2}\right)\right)^{3}}} .
$$

\section{VI.C. One-Component Turbulent Forcing}

One-component vortical gusts with velocity component transverse to the freestream direction, were defined as

$$
v_{1 C}(\boldsymbol{x}, T)=\sum_{l=1}^{L} W_{1 C}\left(\boldsymbol{k}_{l}\right) \cos \left(\boldsymbol{k}_{l}(\boldsymbol{x}-\boldsymbol{U} T)+\sigma\left(\boldsymbol{k}_{l}\right)\right),
$$

where $W_{1 C}$ was obtained by integrating Equation 5 with respect to $k_{y}$ to give 


$$
W_{1 C}\left(k_{x}, 0\right)=\sqrt{\frac{3 \overline{w^{2}} L^{2}}{4 \pi} \frac{L^{2} k_{x}^{2}}{\left(1+L^{2} k_{x}^{2}\right)^{2.5}}} .
$$

\section{VI.D. Discretizing the Energy Spectrum}

Because the computational method uses a discrete number of frequency modes when synthesizing the vortical turbulent input, the continuous spectrum given by Equations 3, 5, and 7 must be appropriately discretized. Therefore, the summation limits $L, M$, and $N$ must be chosen such that the highest $k_{x}, k_{y}$, and $k_{z}$ values, $k_{x}^{l i m}, k_{y}^{\lim }$, and $k_{z}^{l i m}$, can still be resolved by the computational grid.

\section{VI.D.1. Discretising $W_{1 C}$}

$W_{1 C}$ depends on $k_{x}$ only, and therefore requires a distribution of $k_{x}$ to be set. $100 k_{x}$ wavenumbers were defined in the range $0<k_{x}<k_{x}^{l i m}$ where $k_{x}^{l i m}=25 \pi \mathrm{m}^{-1}$. Therefore the highest $k_{x}$ frequency corresponds to a reduced frequency of $K=12.5$ where $K=c / \lambda, c=1 \mathrm{~m}$ is the airfoil chord, and $\lambda$ is the gust wavelength in the streamwise direction. This $k_{x}$ distribution was also used in the discretization of $W_{2 C}$.

\section{VI.D.2. Discretizing $W_{2 C}$}

$W_{2 C}$ contains an additional dependence on $k_{y}$ in comparison to $W_{1 C} \cdot k_{y}^{\text {lim }}$ was chosen to be $100 \mathrm{~m}^{-1}$ This was chosen to provide a compromise between computational cost and full representation of the turbulent spectrum. The distribution of $k_{y}$ was chosen with a greater density around $k_{y}=0$ than at higher values in order to resolve the shape of $W_{2 C}(\boldsymbol{k})$. A total of 46 discrete $k_{y}$ wavenumbers were used. The discretized spectrum contained at least $90 \%$ of the total energy at all $k_{x}$ frequencies. The discretization of $W_{2 C}$ is shown in Figure 2.
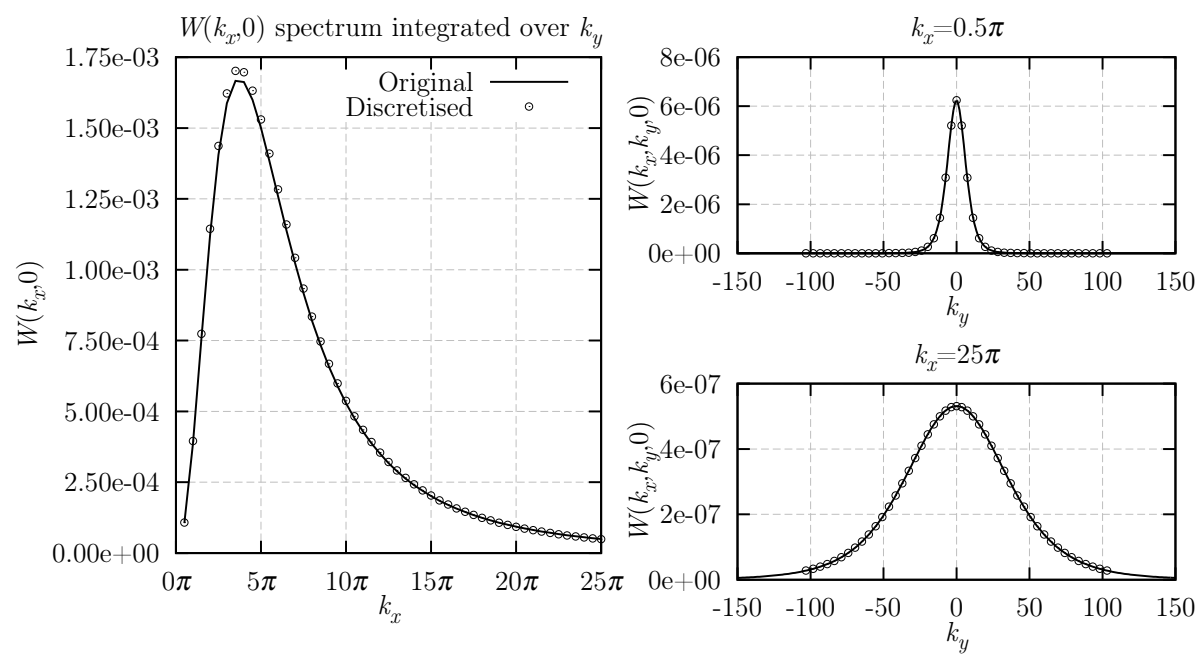

Figure 2. Showing the discretized 2D Liepmann spectrum with varying $k_{x}$ and $k_{y}$.

\section{VI.D.3. Discretizing $W_{3 C}$}

Because the three-component turbulent synthesis method is more computationally expensive than the oneand two-component approaches, a smaller number of wavenumbers was used to discretize $W_{3 C}$. $32 k_{x}$ wavenumbers were defined in the range $0<k_{x}<k_{x}^{\text {lim }}$, where $k_{x}^{\text {lim }}$ was set to $16 \pi \mathrm{m}^{-1}$. $k_{y}^{\text {lim }}$ was also reduced, and set to $75 \mathrm{~m}^{-1}$. $k_{z}^{l i m}$ was chosen to be $50 \mathrm{~m}^{-1}$. The disturbances were required to be periodic about the spanwise width of the simulation. Therefore a spanwise simulation width of $0.5 \mathrm{~m}$ was chosen, and all $k_{z}$ values in the range $-50 \mathrm{~m}^{-1}<k_{z}<50 \mathrm{~m}^{-1}$ were chosen to be harmonics of the fundamental $0.5 \mathrm{~m}$ wavelength. These parameters gave a good representation of the turbulent spectrum while giving an acceptable computational cost. The distribution of $k_{y}$ and $k_{z}$ wavenumbers in the discretized spectrum is 
shown in Figure 3 for $k_{x}=0.5 \pi \mathrm{m}^{-1}$ and $k_{x}=16 \pi \mathrm{m}^{-1}$. The non-discretized spectrum is also shown for comparison.
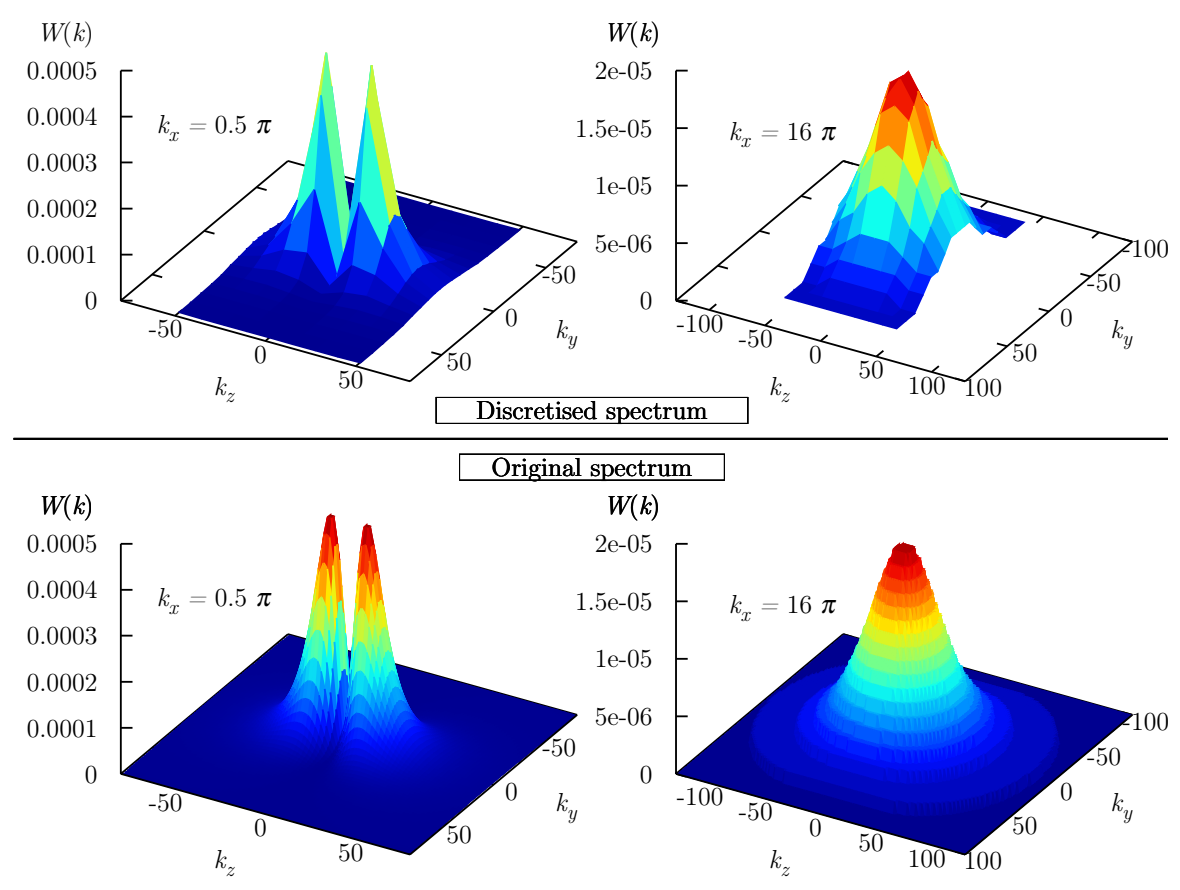

Figure 3. The discretised 3D Liepmann spectrum at low and high values of $k_{x}$.

An example of the one-, two-, and three-component vortical flowfields generated by the turbulent CAA method for one realization, are shown in Figure 4. 

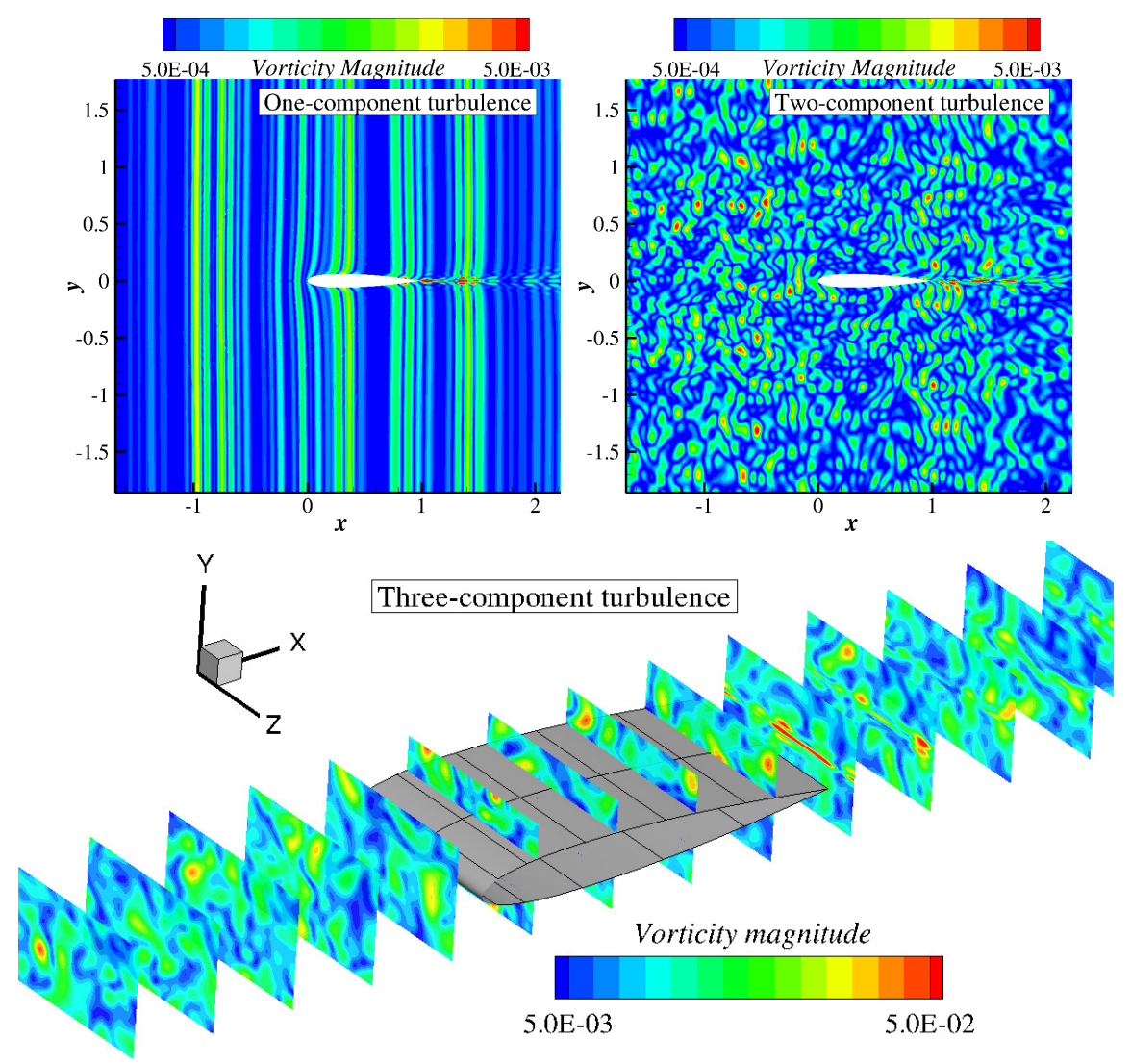

Figure 4. Example flowfield vorticity for the one-, two-, and three-component turbulent synthesis methods. The twoand three-component examples show one realization of the turbulent field. Quantities are non-dimensional.

\section{Tests with Two-Component Turbulent Forcing}

Before a comparison between the three turbulent forcing methods is made, the two-component turbulent synthesis approach is used to study the effects of airfoil geometry on the turbulent interaction noise. A similar study was made in Ref. ${ }^{6}$ using a one-component harmonic gust method. The airfoil thickness tests are repeated using the described two-component synthesis method, to see if the inclusion of the streamwise disturbances affects the turbulent interaction noise for real airfoil geometries.

\section{VII.A. Validation}

In order to validate the approaches used in the current study, the two-component turbulent CAA method was validated by comparing noise predictions from the CAA method with those from Amiet's analytic theory, for the case of a NACA 0002 airfoil encountering upstream turbulence. The turbulent inflow was defined to have $\sqrt{\overline{w^{2}}}=0.04 U_{x}$ and $L=0.07 \mathrm{~m}$, which are comparable turbulence parameters to those used by Amiet. ${ }^{4}$ To give a more accurate comparison between the CAA and analytic predictions, the findings from Ref. ${ }^{6}$ were accounted for, such that the validation was made at a low Mach number, $M=0.2$, used a steady uniform meanflow, and used a thin airfoil with $2 \%$ thickness. These factors ensured that any effects of the airfoil geometry on the results were minimized during the validation study. Figure 5 compares noise predictions from the two-component CAA and analytic methods, at various frequencies and observer angles.

Figure 5 shows agreement between the CAA and analytic methods of better than $2 \mathrm{~dB}$ at all tested frequencies and observer angles. The stochastic "random phase" approach is the cause of the oscillations that are seen in the noise spectra. However, the oscillations are considered sufficiently small because the shape of the noise spectra and the directivity are both captured well by the CAA method. Small disagreements are seen between the CAA and analytic noise predictions at acute upstream angles, where $\theta>150^{\circ}$. This is a result of the real geometry of the NACA 0002 airfoil used in the CAA method, as opposed to the infinitely 
thin flat plate used in the analytic formulation.

\section{VII.B. Comparison with Experiment}

As a further validation of the CAA method used in this paper, the effects on noise due to airfoil geometry were compared with previously measured effects by Paterson and Amiet. ${ }^{28}$ In their experiment, Paterson and Amiet interacted a NACA 0012 airfoil (with chord $c=0.23$ $\mathrm{m}$ and semi-span $d=0.265 \mathrm{~m}$ ) with homogeneous grid-generated turbulence at $M=0.175$, and reported approximately a $5 \mathrm{~dB}$ reduction (compared with analytic flat plate theory) in the noise at $\theta=90^{\circ}$ and at $K_{t}=t / \lambda=1$ (or where $K=c / \lambda=8.3)$. The grid-generated turbulence was characterized by $L=0.13 c$ and $\sqrt{\overline{w^{2}}}=0.039 U_{x}$.

The two-component CAA method was used to predict the noise from the configuration used by Paterson and Amiet. Noise predictions and measurements of the configuration are shown in Figure 6. However, the airfoil span was not accounted for in the CAA predictions because the two-component method is
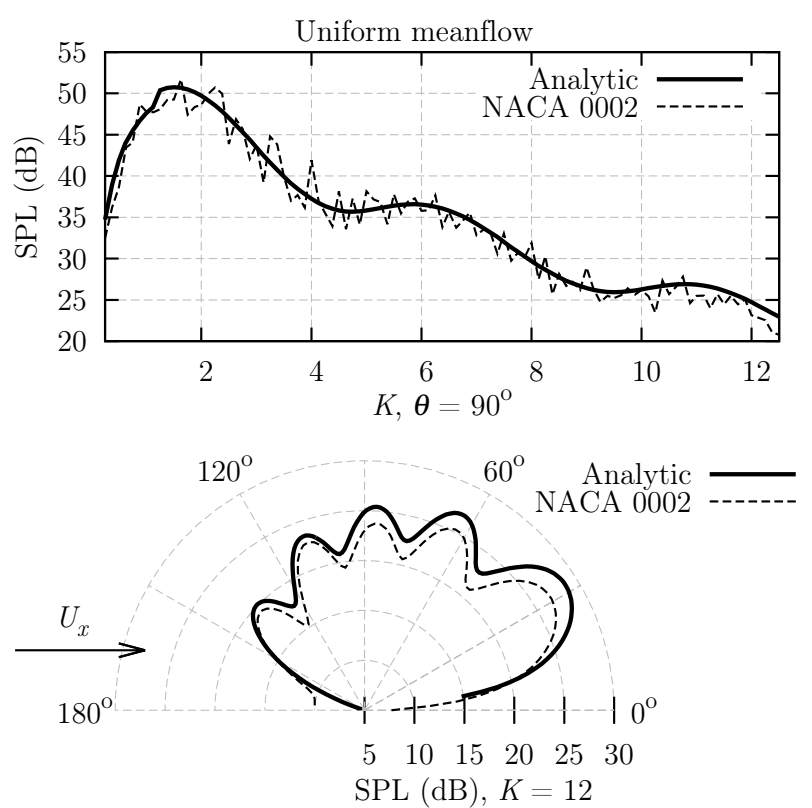

Figure 5. Spectral (upper) and directivity (lower) comparison of CAA and analytic methods for validation. Spectral comparison is made at $\theta=90^{\circ}$ and directivity comparison is made at $K=12$. CAA predictions are made using a NACA 0002 airfoil with a uniform steady meanflow to minimize the effects of airfoil geometry. 2D. Additionally, some operational conditions were not specified by Paterson and Amiet, so $\rho=1.22 \mathrm{~kg} \cdot \mathrm{m}^{-3}$ has been assumed here. Because of the differences between the experimental and computational results, the noise amplitudes are not directly compared in Figure 6. However, the noise reduction in comparison to analytic theory that is caused by airfoil thickness, is directly compared in Figure 7 by defining $\Delta \mathrm{SPL}=\mathrm{SPL}_{\exp }-\mathrm{SPL}_{\text {analytic }}$.

Figure 6 shows a good agreement in terms of spectral shape between the CAA predictions and the measurements. In both methods, the spectral hump that is predicted by analytic theory at $K=6$ and above, appears to be suppressed by the geometry of the NACA 0012 airfoil, such that the noise is reduced for the real airfoil. Figure 7 shows that at $K=8.3$ (or $K_{t}=1$ ), this noise reduction is predicted by the current method, and by Paterson and Amiet, to be slightly greater than $5 \mathrm{~dB}$. Therefore, there is a good agreement between the two studies as to the effects of thickness on turbulent interaction noise.

It initially seems that the turbulent CAA method also agrees with Paterson and Amiet's ${ }^{28}$ conclusion that $K_{t}=1$ (or $K=8.3$ ) can be considered as a measure of when flat plate theory breaks down, because a $5 \mathrm{~dB}$ difference can be seen at $\theta=90^{\circ}$ between CAA and analytical predictions at this frequency in

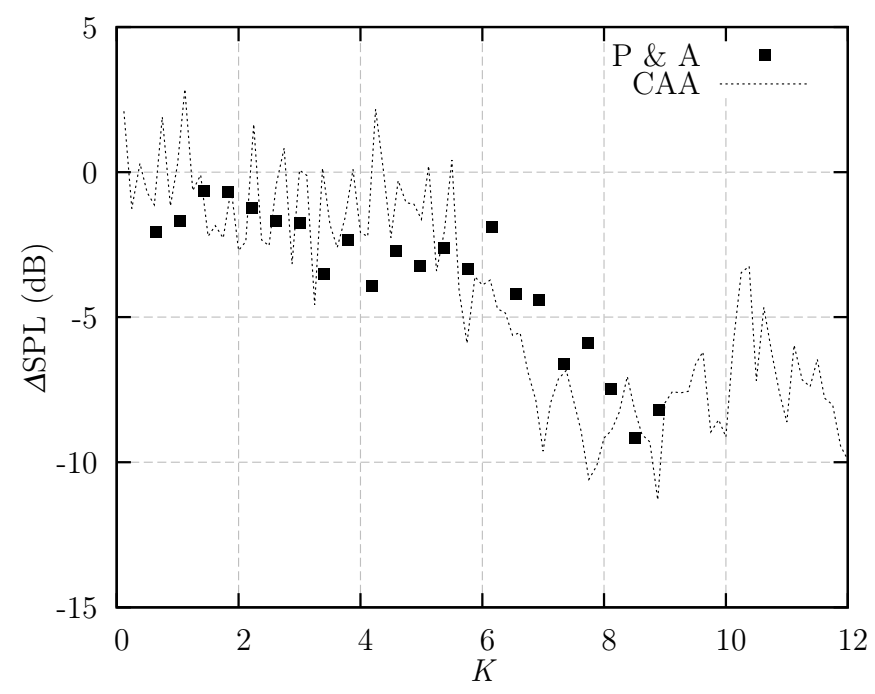

Figure 7. Comparison of $\triangle \mathrm{SPL}$ due to the thickness of a NACA 0012 airfoil in $M=0.175$ flow, measured by Paterson and Amiet $^{28}$ and predicted by the current CAA method.

Figure 6. However, this comparison does not consider all observer angles. Figure 8 compares PWL predictions from the CAA and analytic methods. This 

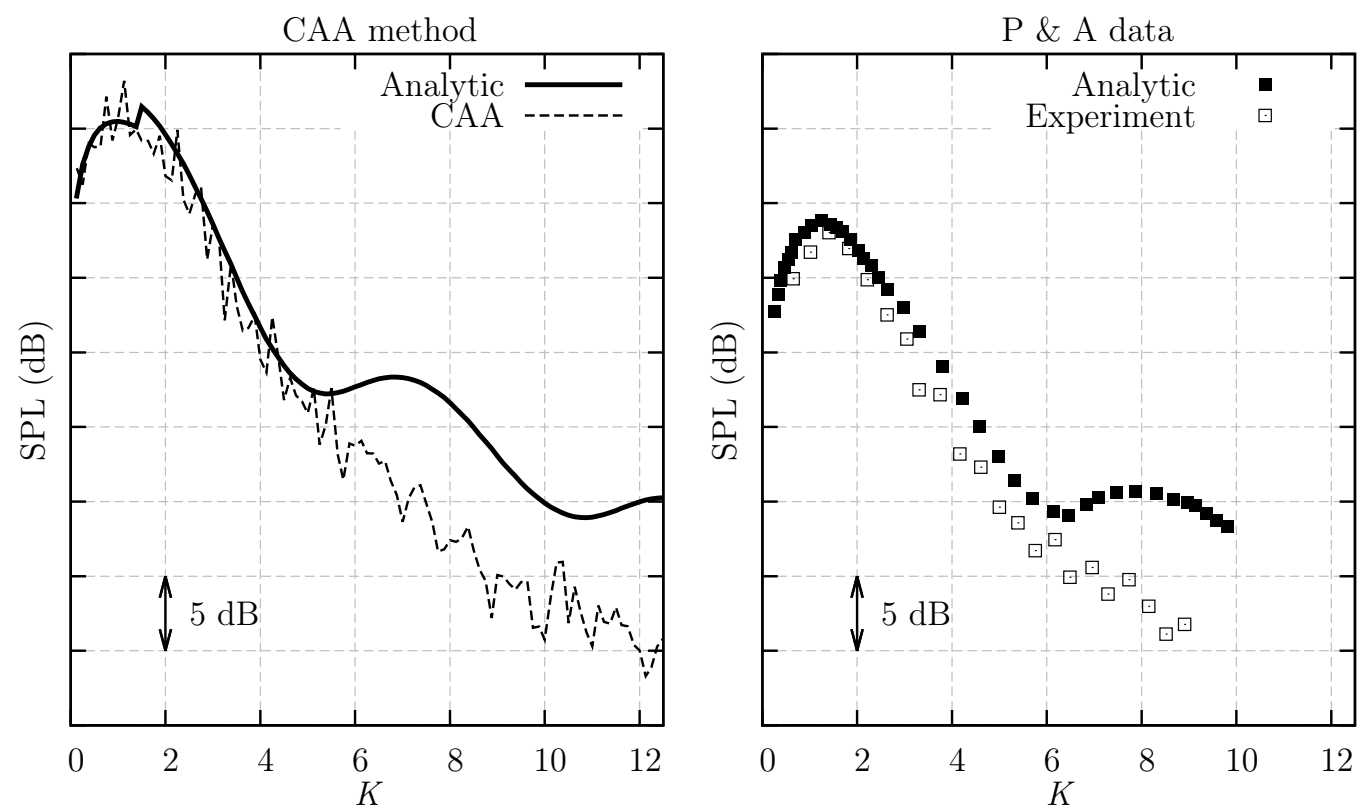

Figure 6. Left compares between predictions made by the two-component CAA method and by analytic flat plate theory. Right compares between experimental measurements and analytic predictions that were made by Paterson and Amiet. $^{28}$

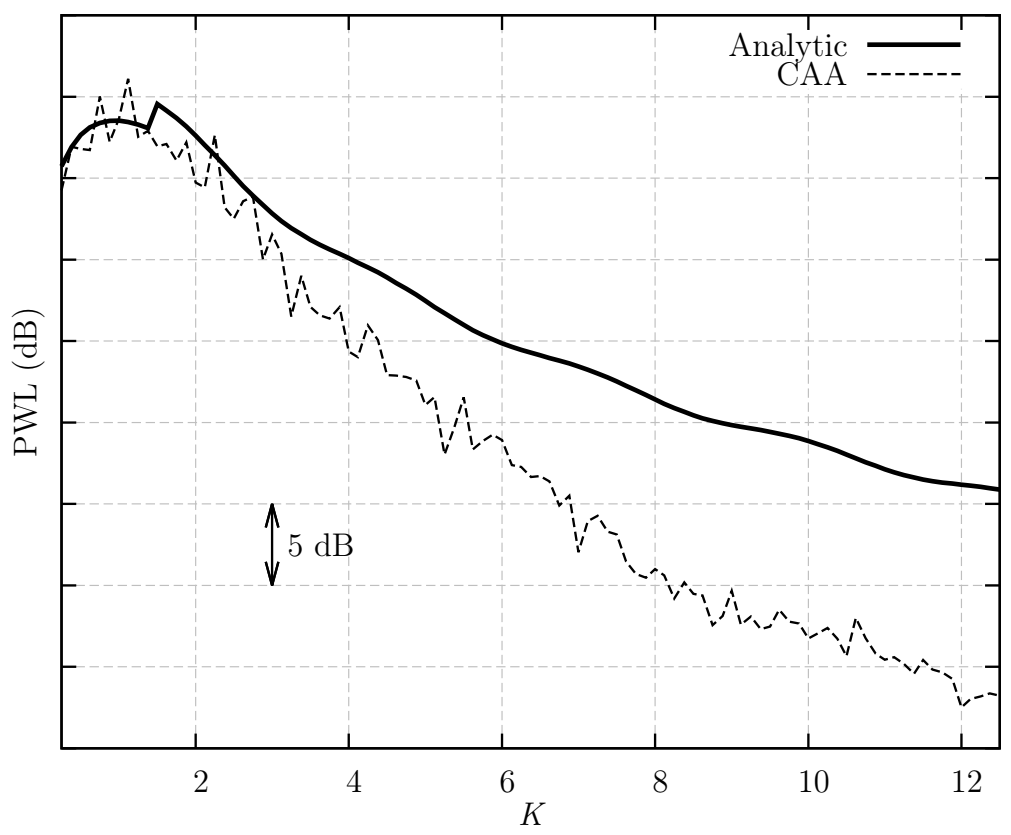

Figure 8. Comparison of PWL predictions made by analytic theory and by the two-component CAA method for a NACA 0012 airfoil in $M=0.175$ flow. 
comparison shows that once the effects at all observer angles are accounted for, the effects of thickness on turbulent interaction noise occur at about $K=3$. Therefore, flat plate theory can be considered to break down at about $K=3$ in this case, which is a lower value than suggested by Paterson and Amiet. Note that since Paterson and Amiet provide spectral data only at $\theta=90^{\circ}$ their results are not included in Figure 8.

The successful validation of the CAA method against both experimental measurement and analytical modeling gave good confidence of the CAA noise predictions.

\section{VII.C. Effects of Airfoil Thickness on Turbulent Interaction Noise}

The effects of airfoil thickness on turbulent interaction noise were tested on a range of airfoils using the two-component turbulent forcing method. Eleven NACA 4-digit airfoils were used, with thicknesses ranging from $2 \%$ to $12 \%$. The tested airfoils are shown in Figure 9. All tests in this section were conducted at $M=0.5$.

To gain an overall view of the effects of airfoil thickness geometry, Figure 10 shows contours of SPL predictions from the analytic flat plate model and from the two-component turbulent CAA method using airfoils with $4 \%, 8 \%$ and $12 \%$ thickness. In each case, the variation of SPL with both $\theta$ and $K$ is shown so that the full implications of airfoil geometry on the noise can be seen. Figure 10 shows that at high reduced frequencies, SPL predictions for thick airfoils are reduced in comparison to flat plate theory, and that this reduction increases with increasing thickness. It also shows that as the airfoil thickness is increased, SPL predictions become more constant with observer angle $\theta$. Analytic predictions are clearly louder

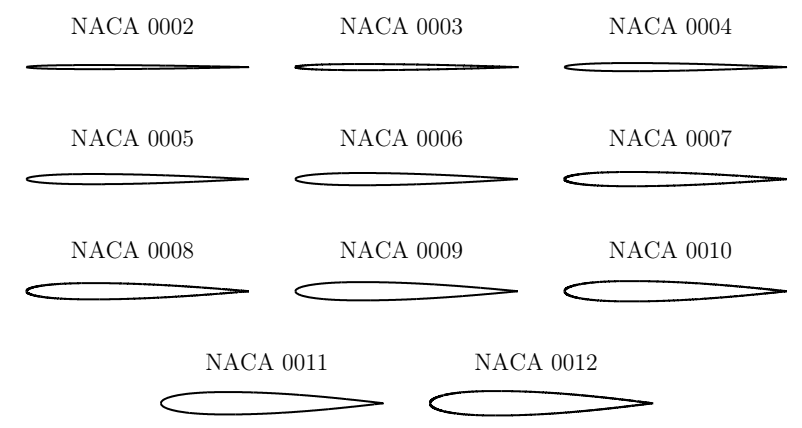

Figure 9. The various airfoils used in the study. The thickness of NACA 4-series airfoils was varied between $2 \%$ and $12 \%$. at $\theta=20^{\circ}$ compared with $\theta=180^{\circ}$, whereas there is little difference between SPL results at these two angles for the NACA 0012 case. Figure 10 also shows that the oscillations which are seen in the analytic solution due to interactions between the leading and trailing edges, become more smoothed as the airfoil thickness is increased. These oscillations are still present for the NACA 0004 case but are no longer observable for the NACA 0012 case.

In order to better visualize the noise reduction that is caused by airfoil geometry, contours of $\Delta$ SPL are plotted in Figure 11 for the $4 \%, 8 \%$ and $12 \%$ airfoils. $\Delta$ SPL was calculated as the difference in noise between each CAA case and the analytic flat plate prediction, such that $\Delta \mathrm{SPL}(\theta, K)=\mathrm{SPL}_{c a a}(\theta, K)-$ $\operatorname{SPL}_{\text {analytic }}(\theta, K)$. The scale of the contours shown in Figure 11 is limited to only show areas where a reduction in noise was observed in comparison to the analytic prediction. Figure 11 shows that at downstream observer angles $\left(\theta<90^{\circ}\right)$ there is a reduction in the noise at most frequencies, whereas upstream observer angles only experience a noise reduction at higher values of reduced frequency. Additionally, the reduction is greater in the downstream direction than in the upstream direction, as was observed previously in Ref. ${ }^{6}$ and by Atassi et al. ${ }^{14}$

To quantify the effects on leading edge noise due to airfoil geometry when using synthesized turbulence, Figure 12 compares PWL spectra and SPL directivity plots of airfoils with $4 \%, 8 \%$ and $12 \%$ thickness. The PWL spectra in Figure 12 shows that the reduced frequency at which leading edge noise becomes sensitive to airfoil geometry is at about $K=5$. The noise prediction for the NACA 0012 case at $K=12$ is about 11 $\mathrm{dB}$ lower than the analytic noise prediction. Figure 12 also shows that the general shape of the directivity pattern appears to be unchanged by the presence of airfoil thickness.

Figure 12 also shows that the noise, in terms of Decibels, does not seem to reduce linearly with increasing thickness. At $K=12$, there is very little noise reduction shown by the NACA 0004 case, while about $5 \mathrm{~dB}$ is shown by the NACA 0008 case, and about $11 \mathrm{~dB}$ is shown by the NACA 0012 case. Previous studies, such as those by Olsen and Wagner, ${ }^{8}$ and Roger and Moreau, ${ }^{10}$ have reported an apparent linear decrease in noise with increasing airfoil thickness. To explain the discrepancy between previous studies and the current work, the variation of both sound power $P$, and PWL, with increasing airfoil thickness $t$, is shown in Figure 13 at two reduced frequencies. Figure 13 shows that at constant reduced frequency, the sound power $P$ appears 

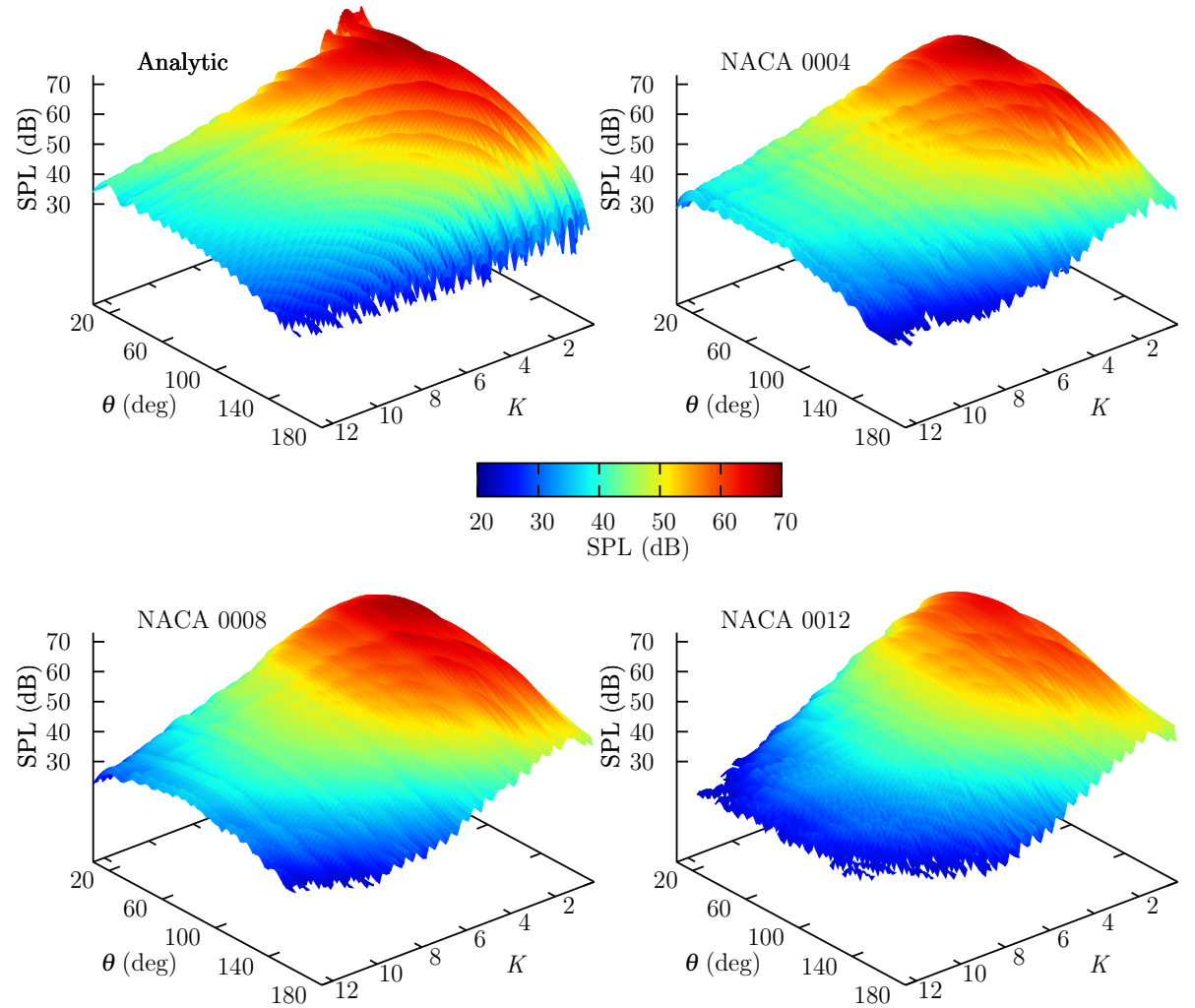

Figure 10. Contours of SPL with varying observer angle $\theta$ and reduced frequency $K$ for the analytic method, compared with CAA predictions using a $4 \%, 8 \%$, and $12 \%$ airfoil.

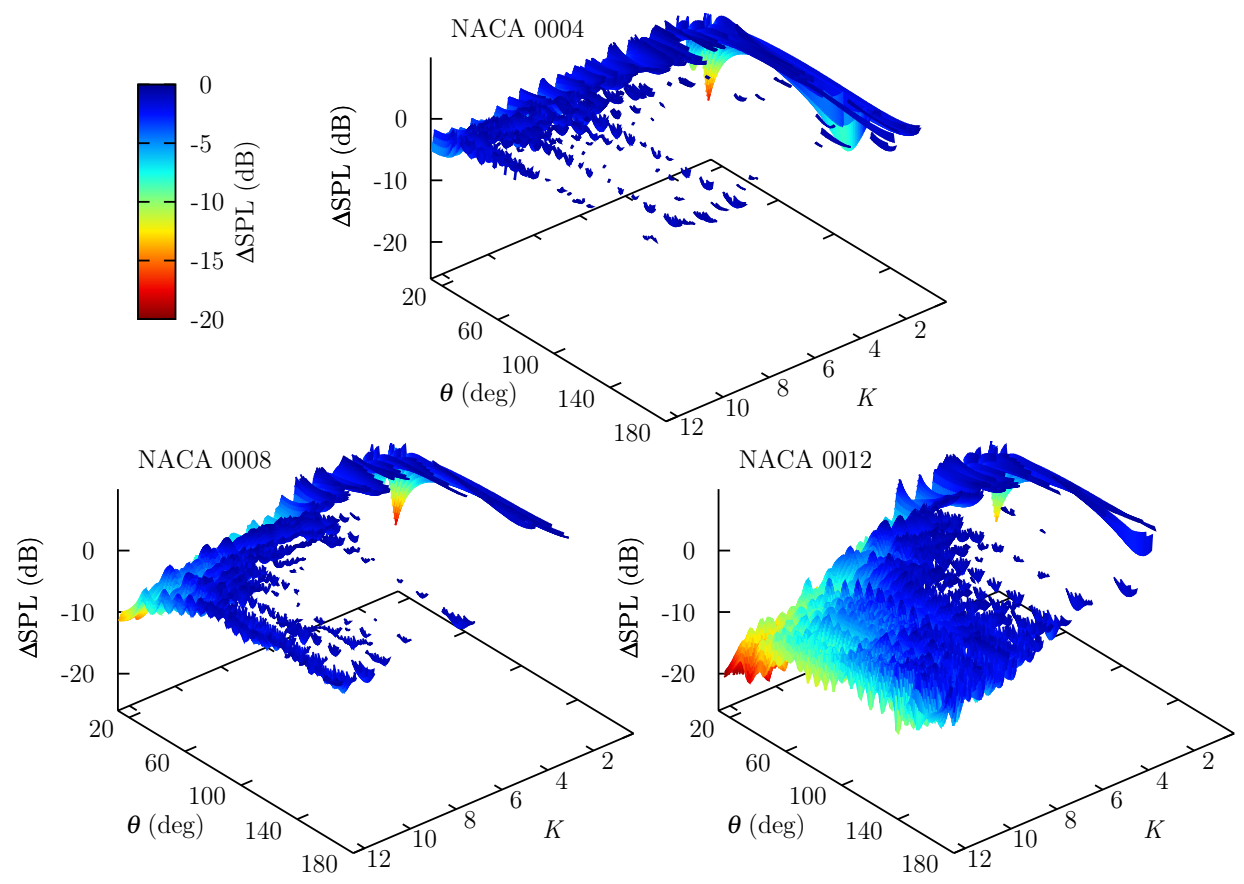

Figure 11. $\triangle S P L$ with varying observer angle $\theta$ and reduced frequency $K$ against the baseline flat plate prediction, for a $4 \%, 8 \%$ and $12 \%$ thick airfoil. 

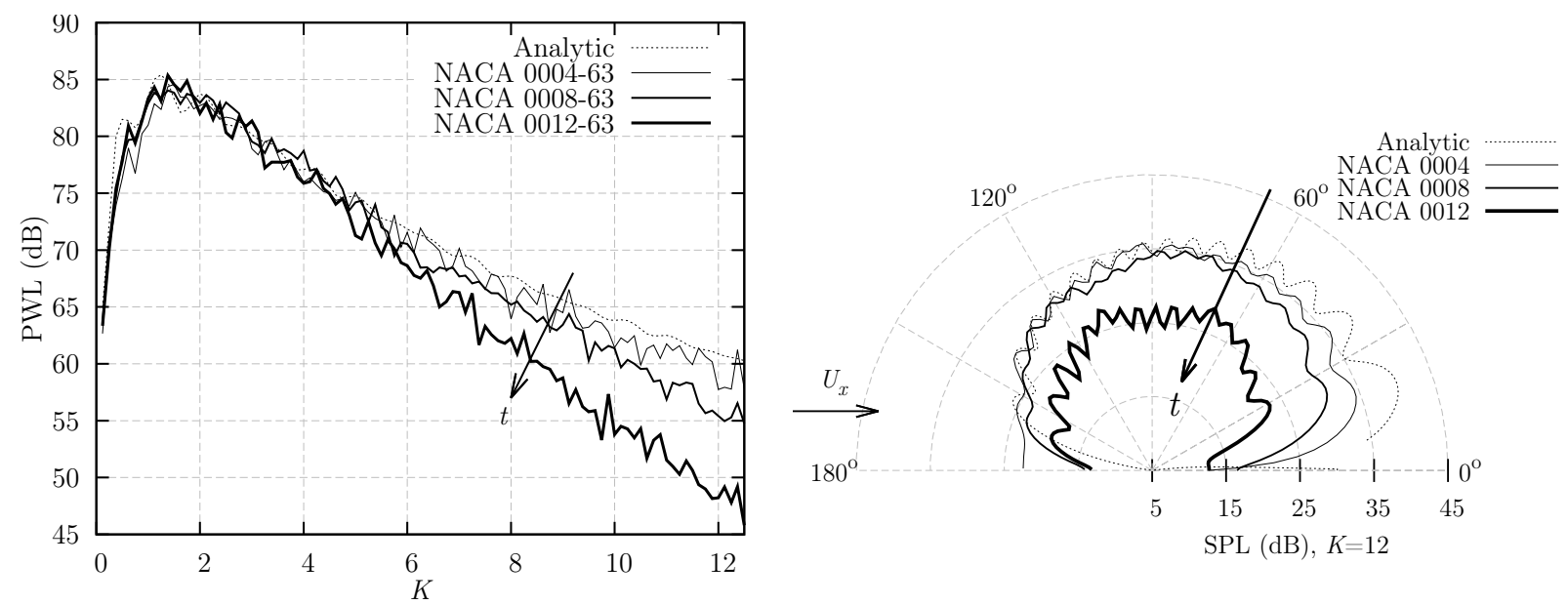

Figure 12. CAA PWL (left) and directivity (right) predictions for a $4 \%$, $8 \%$, and $12 \%$ airfoil compared with analytic theory.

to reduce linearly with increasing thickness, whereas PWL reduces logarithmically as would be expected. This explains the noise behavior with increasing thickness that is seen in Figure 12. The linear relationship between $P$ and $t$ was reported previously in Ref., ${ }^{6}$ but it is confirmed here with the use of a larger number of airfoil thicknesses and with a two-component turbulent forcing method. This relationship appears to hold even for $2 \%$ thick airfoils.
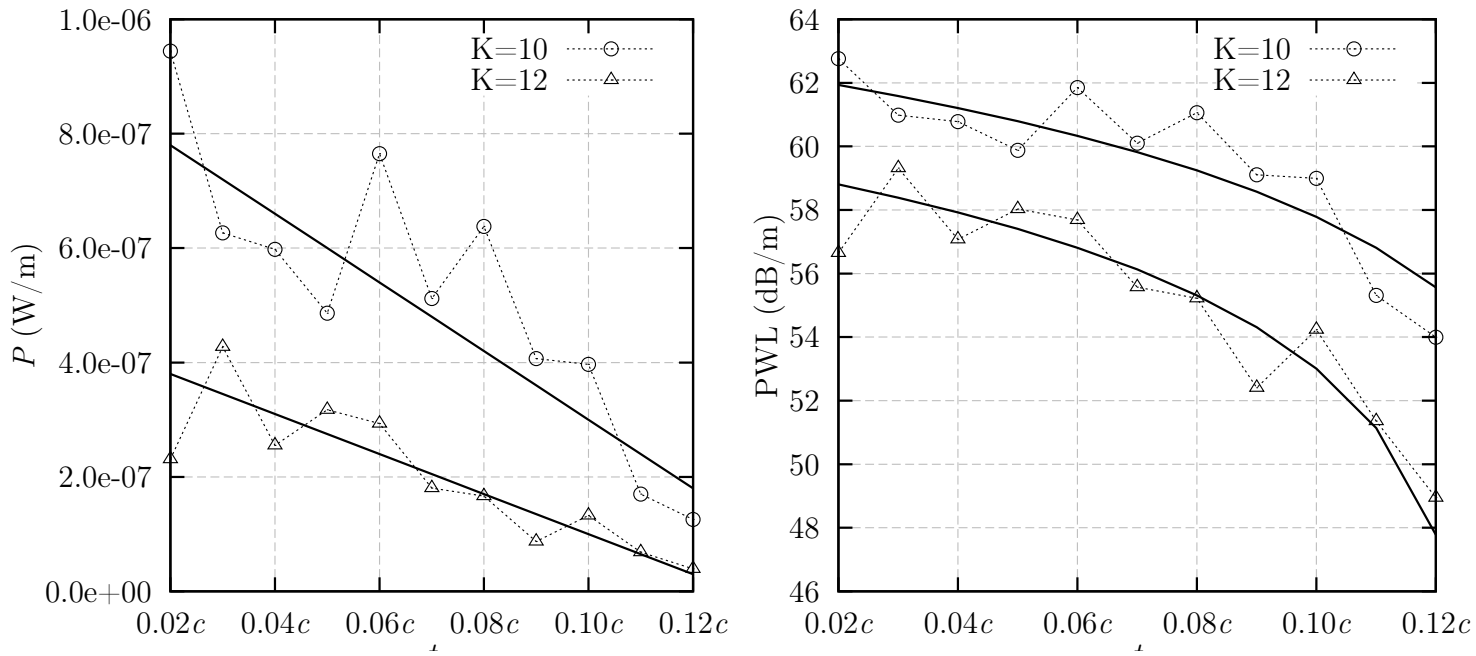

Figure 13. Sound power $P$ and sound power level (PWL) variation for airfoils with various thicknesses at $K=10$ and $K=12$. Solid lines are drawn as lines of best fit.

It has been observed that for $M=0.5$ flow, the threshold reduced frequency above which airfoil thickness will affect the PWL predictions is about $K=5$. In Section VII.B this frequency was observed to be about $K=3$ in $M=0.175$ flow. Therefore, it appears that this threshold frequency has some dependence on the Mach number, such that the threshold frequency will increase with increasing Mach number. A study to fully quantify this effect would be useful. 


\section{VII.D. Mechanism of Noise Reduction}

To determine whether the mechanism that causes the reduced noise for thick airfoils and two-component forcing, is similar to the findings in Ref., ${ }^{6}$ contours of the root-mean-square velocity perturbations around the airfoil leading edge are plotted for a $2 \%, 6 \%$ and $12 \%$ thick airfoil in Figure 14 . Because the twocomponent CAA method uses gusts with both transverse and streamwise velocity perturbations, Figure 14 shows contours of $U_{r m s}$ where $U_{r m s}=\sqrt{u_{r m s}^{2}+v_{r m s}^{2}}$. An ensemble average was taken of the flowfield from multiple realizations in order to provide the root-mean-square values shown.

Figure 14 shows that the $U_{r m s}$ values reach a peak value at the leading edge of the NACA 0002 airfoil, but that this peak is weaker for the thicker NACA 0006 and NACA 0012 airfoils. This finding is consistent with Ref. ${ }^{6}$ which used transverse gust forcing, and suggests that the mechanism for noise reduction is the same whether one- or two-component gust forcing is used. Reductions in noise are caused by the distortion of high frequency vortical disturbances, such that the amplitude of the disturbances at the leading edge is reduced. The distortion is caused by velocity gradients in the meanflow field. The reduced vortical disturbances then provoke a reduced pressure response when they interact with the airfoil surface, and therefore generate less noise.
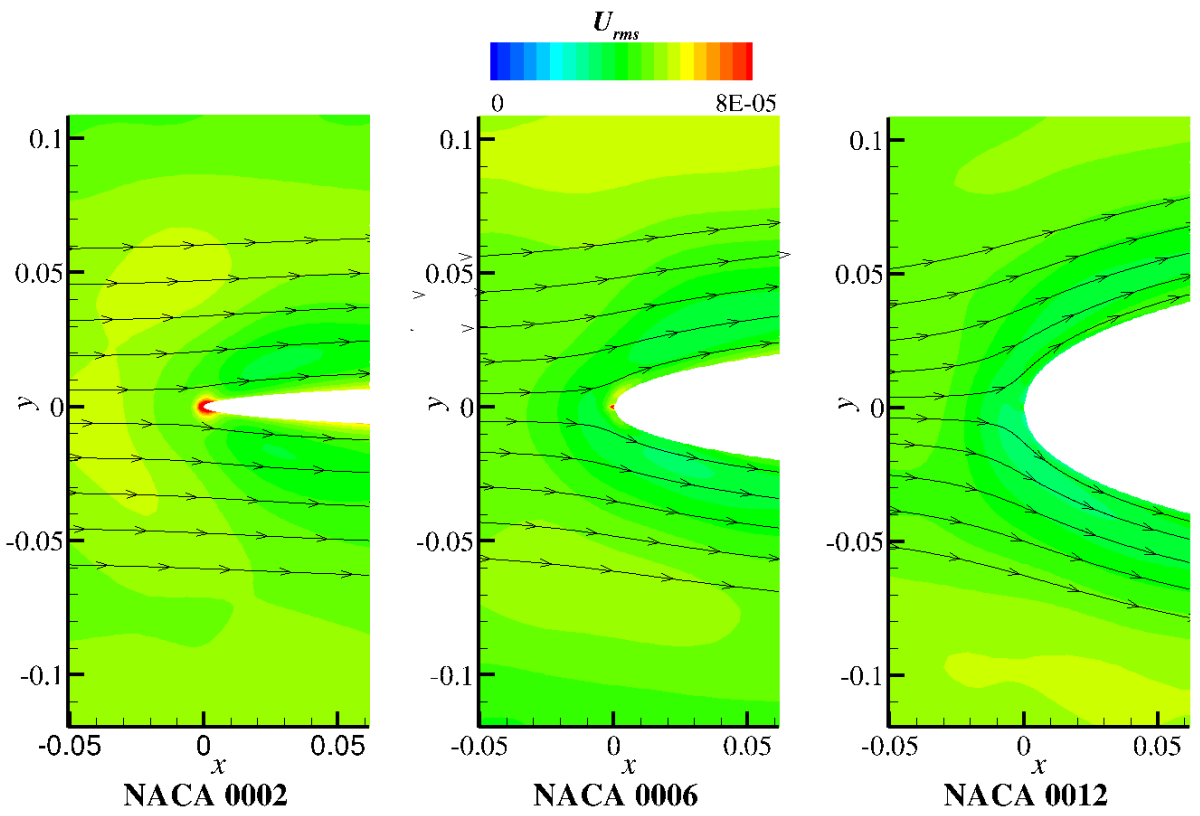

Figure 14. Contours of $U_{r m s}$ for gusts with $K=8$ and various $k_{y}$ wavenumber components interacting with 2\%, 6\%, and $12 \%$ thick airfoils. The contours are overlaid with streamlines of the non-uniform meanflow around each airfoil. Quantities are non-dimensional. 


\section{Reduced Dimension Modeling}

Thus far, the behavior of turbulent interaction noise with increasing airfoil thickness appears to be similar to the predictions found in Ref. ${ }^{6}$ which used a one-component transverse gust forcing method. This suggests that the addition of streamwise disturbances may be unnecessary when modeling the turbulent interaction noise of symmetric airfoils at zero angle of attack, and that the transverse disturbances are dominant.

As discussed in Section III, Amiet ${ }^{29}$ also highlighted the importance of the transverse disturbances, and showed that for flat plates with $d>>L$, only transverse gusts whose spanwise wavenumber is $k_{z}=0$ need to be modeled.

If Amiet's simplification is also valid for real airfoils, it could be combined with the findings from the current work to show that only the transverse turbulent component is important when modelling turbulent interaction noise. Significant reductions in computational time would be achieved if 3D constant span airfoils can be accurately modeled by using only transverse velocity disturbances.

This Section will predict the noise from a NACA 0012 airfoil in $M=0.5$ flow, by using the one-, two-, and three-component turbulent forcing methods discussed in Section VI. Comparisons between the three noise predictions will highlight if there are any effects on the noise due to the inclusion of streamwise and spanwise disturbances. The three-component method requires a three-dimensional simulation and is very computationally expensive. Therefore, this reduced dimension modeling test will only be conducted on one airfoil geometry.

\section{VIII.A. Computational Efficiency}

Several techniques were used in order to improve the computational efficiency of the three-component forcing method:

- Reduced cell count. The $k_{x}^{\text {lim }}$ and $k_{y}^{\text {lim }}$ values were redefined in order to permit a smaller computational grid. $k_{x}^{\text {lim }}=16 \pi \mathrm{m}^{-1}$ and $k_{y}^{\text {lim }}=75 \mathrm{~m}^{-1}$ were used in the one-, two-, and three-component models to allow a close comparison between the different approaches.

- Spanwise periodicity. Periodic boundary conditions were applied on the two spanwise faces of the simulation in order to simulate an infinite span airfoil. This reduced the computational expense by avoiding the complex geometry at the wing tips, and also made the $3 \mathrm{D}$ simulation more comparable to the $2 \mathrm{D}$ simulations. This simplification required the three-component synthesized turbulence to also be periodic in the spanwise direction. Therefore, the largest spanwise wavelength was set equal to the width of the domain.

- Reduced downstream resolution. The wavelength of the vortical waves was smaller than the wavelength of the acoustic waves. This allowed the resolution of the grid to be reduced downstream of the airfoil. In this region, the vortical gusts do not need to be fully resolved since they have already interacted with the airfoil surface. Instead, the resolution requirement downstream of the airfoil was set to resolve only the acoustic waves, which allowed for a reduction in the total cell count. The final cell count of the $3 \mathrm{D}$ computational grid was approximately 26 million cells. The $2 \mathrm{D}$ computational grid consisted of approximately 400, 000 cells.

- Improved load balancing. A large number of processors was required for the 3D simulation in order to obtain solutions in a reasonable time. The CAA method typically balances the load across multiple processors by ensuring that each processor is assigned a similar number of cells. However, when the three-component turbulent synthesis was activated, this balancing technique was less effective because the turbulent synthesis created additional expense in the buffer regions. Therefore, the load balancing was altered to reduce the cell count of any processor involved in the generation of the synthetic turbulence.

\section{VIII.B. Comparison of One-, Two-, and Three-Component Methods}

The far-field noise was predicted by the CAA method, using one-, two-, and three-component turbulent interactions with a NACA 0012 airfoil. A comparison of the SPL noise spectra predictions from each approach, and from Amiet's analytic theory, is shown in Figure 15. 

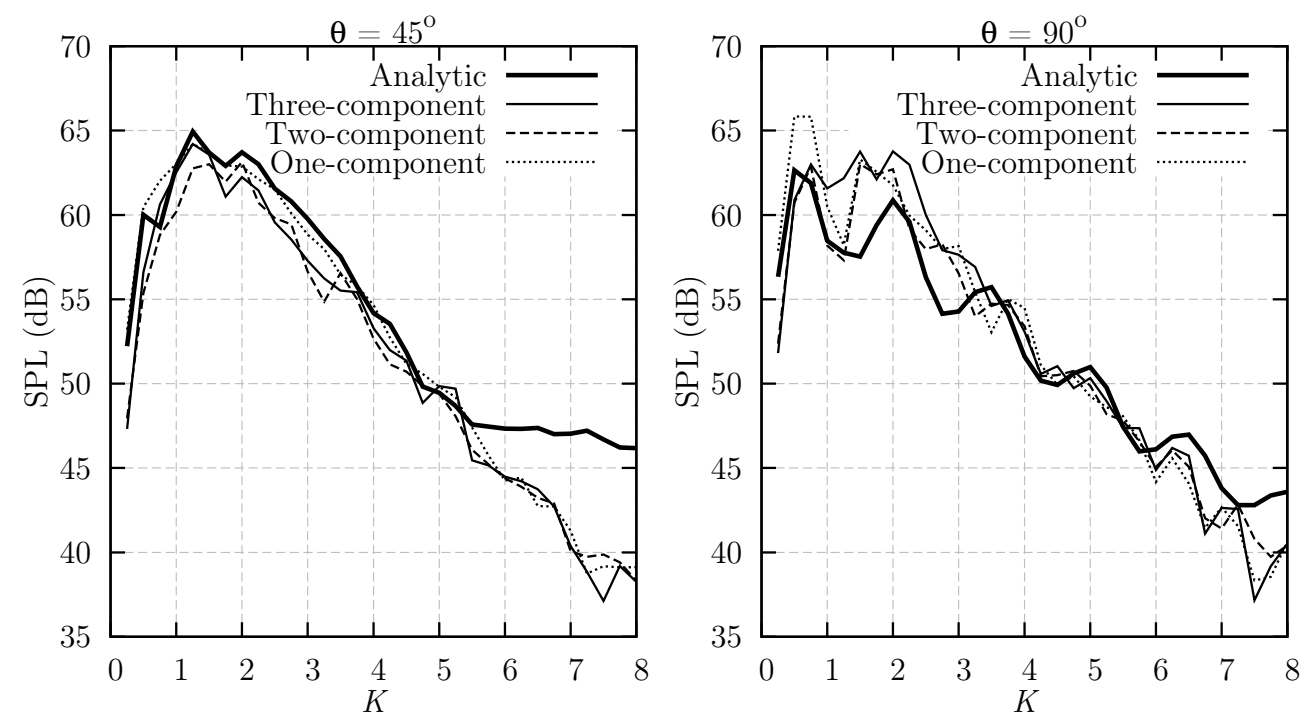

Figure 15. SPL Predictions at observer positions of $\theta=45^{\circ}$ (left) and $\theta=90^{\circ}$ (right) due to a NACA0012 airfoil interacting with one-, two-, and three-component turbulence in $M=0.5$ flow, compared with Amiet's analytic theory.

Figure 15 shows that very similar noise predictions are given by the one-, two-, and three-component turbulent synthesis methods, at both of the observer angles studied. There are some differences shown by the one-component method, which predicts a higher sound than the other methods below $K=2$. However this difference is usually less than $1 \mathrm{~dB}$ and is considered small. When $K<2$, the $k_{y}$ gust components generally have large wavelengths in relation to the airfoil thickness. Therefore, the differences shown by the one-component method may be caused by the assumption that all $k_{y}$ wavenumbers interact with the airfoil, whereas in reality some components are sufficiently large in comparison to the airfoil thickness that they do not generate noise.

Figure 16 shows a comparison of the PWL noise and the SPL directivity from each turbulent synthesis method. Close agreement is seen between the three methods for PWL predictions and for SPL directivity predictions. In all three methods, the PWL prediction is approximately $8 \mathrm{~dB}$ lower than the analytical method due to the thickness of the NACA 0012 airfoil. This reduction is also shown by all three methods to be greater for downstream observers than for upstream ones.
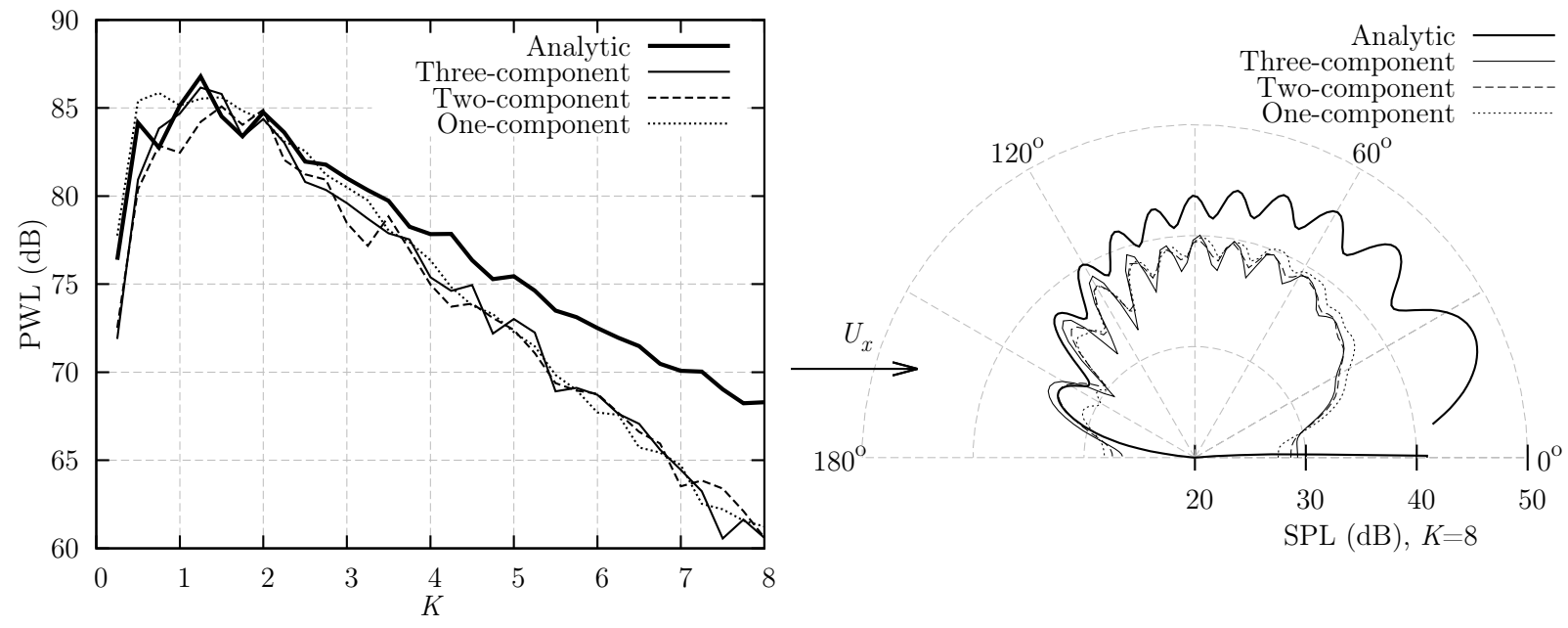

Figure 16. PWL (left) and SPL directivity (right) predictions due to a NACA0012 airfoil interacting with one-, two-, and three-component turbulence in $M=0.5$ flow, compared with Amiet's analytic theory.

The close agreement between predictions made using the one-, two-, and three-component turbulent forc- 
ing methods suggests that transverse disturbances are the dominant noise generating turbulent component, and that accurate noise predictions can be made by using only transverse disturbances. The addition of streamwise and spanwise disturbances has little effect on noise predictions. As previously discussed, the relative unimportance of spanwise disturbances for cases where $d>>L$ was originally suggested by Amiet for flat-plates, but the result has been reproduced here for generic airfoil profiles. In terms of the velocity amplitude spectrum $W(\boldsymbol{k})$, this means that the full spectrum $W_{3 C}\left(k_{x}, k_{y}, k_{z}\right)$ can be simplified to $W_{1 C}\left(k_{x}, 0\right)$ without significant loss of noise prediction accuracy. This simplification is done by setting $k_{z}=0$ and by integrating over all $k_{y}$ wavenumbers.

The incentive to use a reduced dimension model is to reduce the computational cost. Therefore it is useful to compare the relative computational expenses of the three methods. Table 1 compares the computational expense needed for the one-, two-, and three-component turbulent methods. The three cases were each run in the SotonCAA code using the Iridis4 High Performance Computing Facility at the University of Southampton.

A single parameter was desired to allow the total expense of the three simulations to be compared. Therefore, in Table 1, the total expense is calculated as total expense $=$ time for 1 realization $\times$ no. realizations $\times$ number of cores.

\begin{tabular}{|l|l|l|l|l|l|}
\hline Method & No. cells & $\begin{array}{l}\text { No. } \\
\text { cores }\end{array}$ & $\begin{array}{l}\text { Time for one re- } \\
\text { alization }(\mathbf{s})\end{array}$ & $\begin{array}{l}\text { No. realiza- } \\
\text { tions }\end{array}$ & $\begin{array}{l}\text { Total expense } \\
\text { (s) }\end{array}$ \\
\hline $\begin{array}{l}\text { One- } \\
\text { component }\end{array}$ & 400,000 & 16 & 3,716 & 1 & 59,456 \\
\hline $\begin{array}{l}\text { Two- } \\
\text { component }\end{array}$ & 400,000 & 16 & 4,053 & 20 & $1,296,000$ \\
\hline $\begin{array}{l}\text { Three- } \\
\text { component }\end{array}$ & $26,000,000$ & 192 & 77,725 & 20 & $298,464,000$ \\
\hline
\end{tabular}

Table 1. Comparison of computational expense of the one-, two-, and three-component models.

A large difference in expense is seen between the three methods. The three-component method shows the greatest expense due to the significantly larger computational grid that is required. The one-component method makes cost savings by having a smaller computational grid, and also by not requiring any separate realizations for time-decorrelation. The result is that the one-component method is approximately 5,000 times cheaper to compute than the three-component method.

The difference in computational expense between one- and three-component simulations may be less extreme if an alternative turbulence synthesis method were used. Some of the additional expense in the three-component method is due to the summation over $l, m$, and $n$ modes, which is only necessary for turbulent synthesis via a summation of Fourier modes. However, testing the computational expense of alternative synthesis methods is beyond the scope of the current study. Additionally, a smaller number of realizations could be used in the two-, and three-component methods in order to reduce their computational time. For example, Clair et al. ${ }^{16}$ used 10 realizations.

\section{Summary}

Noise that is generated due to interactions between an oncoming turbulent flow and a real airfoil, has been modeled by previous authors using a variety of experimental, analytical and computational methods. However, previous studies made using computational methods have been limited to modeling one-component transverse vortical gusts, which is a simplification of turbulent flow.

The current work has used a CAA method to synthesize a one-, two-, and three-component turbulent inflow (including transvers, streamwise and transverse, and streamwise, transverse, and spanwise distrubances, respectively), in order to model leading edge turbulent interaction noise for real symmetric airfoils. The twocomponent turbulent synthesis method, which models a homogeneous and isotropic turbulent disturbance with streamwise and transverse components, has been used to test the effects of symmetric airfoil thickness on the turbulent interaction noise. It has been found that increasing the airfoil thickness will reduce the noise at high frequencies, particularly for downstream observer angles, and that for a constant reduced frequency the sound power $P$ will reduce linearly with airfoil thickness. These findings are similar to previous studies which used a one-component turbulent synthesis method. This suggests that the addition of streamwise 
turbulent disturbances has little effect on the noise.

The airfoil geometry study has also highlighted that the threshold reduced frequency, above which the airfoil geometry will affect the noise, is lower than was predicted by Paterson and Amiet ${ }^{28}$ when all the observer angles are accounted for. This threshold frequency also has some dependence on Mach number.

One-, two-, and three-component turbulent forcing methods are outlined in the current work, and each has been used to compute the noise from a NACA 0012 airfoil interacting with oncoming turbulence in $M=0.5$ flow. It was found that all three methods give similar noise predictions for this case. Therefore, this analysis suggests that the streamwise and spanwise turbulent disturbances are relatively unimportant to the noise, and that accurate predictions can be made by only modeling the transverse turbulent disturbances. This simplification of the turbulent inflow allows significant computational savings to be made. In the current study, the one-component method can provide noise predictions approximately 5,000 times faster than the three-component approach.

This analysis is currently limited to modeling the leading edge turbulent interaction noise from symmetric constant span airfoils at zero angle of attack, and whose span is significantly larger than the turbulent integral lengthscale. Testing the accuracy of one-component turbulence modeling methods for real airfoils with nonzero angle of attack and camber, and varying spanwise cross-section, would provide interesting future study. 


\section{References}

1 "Flightpath 2050 Europe's Vision for Aviation: Report of the High Level Group Research," European Commission, 2011.

${ }^{2}$ Sears, W. J., "Some Aspects of Non-Stationary Airfoil Theory and its Practical Applications," Journal of Aerospace Sciences, Vol. 8, 1941, pp. 104-108.

${ }^{3}$ Graham, J. M., "Similarity Rules for Thin Aerofoils in Non-Stationary Subsonic Flows," Journal of Fluid Mechanics, Vol. 43, No. 4, 1970, pp. 753-766.

${ }^{4}$ Amiet, R. K., "Acoustic Radiation from an Airfoil in a Turbulent Stream," Journal of Sound and Vibration, Vol. 41, No. 4, 1975, pp. 407-420.

${ }^{5}$ Devenport, W. J., Staubs, J. K., and Glegg, S. A. L., "Sound Radiation from Real Airfoils in Turbulence," Journal of Sound and Vibration, Vol. 329, 2010, pp. 3470-3483.

${ }^{6}$ Gill, J., Zhang, X., and Joseph, P., "Symmetric Airfoil Geometry Effects on Leading Edge Noise," Journal of the Acoustic Society of America, Vol. 134, No. 4, 2013, pp. 2669-2680.

${ }^{7}$ Paterson, R. W. and Amiet, R. K., "Acoustic Radiation and Surface Pressure Response of an Airfoil due to Incident Turbulence," Tech. Rep. CR-2733, NASA, 1976.

${ }^{8}$ Olsen, W. and Wagner, J., "Effect of Thickness on Airfoil Surface Noise," AIAA Journal, Vol. 20, No. 3, 1981, pp. 437439.

${ }^{9}$ Hall, A. M., Atassi, O., and Gilson, J., "Effects of Leading-Edge Thickness on High-Speed Airfoil-Turbulence Interaction Noise," 17th AIAA/CEAS Aeroacoustics Conference, Portland, Oregon, No. 2011-2861, 2011.

${ }^{10}$ Roger, M. and Moreau, S., "Extensions and Limitations of Analytical Airfoil Broadband Noise Models," International Journal of Aeroacoustics, Vol. 9, 2010, pp. 273-305.

${ }^{11}$ Gershfeld, J., "Leading Edge Noise from Thick Foils in Turbulent Flow," Journal of the Acoustical Society of America, Vol. 116, No. 3, 2004, pp. 1416-1426.

${ }^{12}$ Glegg, S. A. L. and Devenport, W. J., "Panel Methods for Airfoils in Turbulent Flow," Journal of Sound and Vibration, Vol. 329, 2010, pp. 3709-3720.

${ }^{13}$ Moreau, S., Roger, M., and Jurdic, V., "Effect of Angle of Attack and Airfoil Shape on Turbulence Interaction Noise," 11th AIAA/CEAS Aeroacoustics Conference, Monterey, California, No. 2005-2973, 2005.

${ }^{14}$ Atassi, H. M., Subramaniam, S., and Scott, J. R., "Acoustic Radiation from Lifting Airfoils in Compressible Subsonic Flow," Tech. Rep. AIAA-90-3911, NASA, 1990.

${ }^{15}$ Lockard, D. and Morris, P., "Radiated Noise from Airfoils in Realistic Mean Flows," AIAA Journal, Vol. 36, No. 6, 1998, pp. 907-914.

${ }^{16}$ Clair, V., Polacsek, C., Garrec, T. L., Reboul, G., Gruber, M., and Joseph, P., "Experimental and Numerical Investigation of Turbulence-Airfoil Noise Reduction Using Leading Edge Serrations," 18th AIAA/CEAS Aeroacoustics Conference, Colorado Springs, No. AIAA 2012-2189, 2012.

${ }^{17}$ Evers, I. and Peake, N., "On Sound Generation by the Interaction Between Turbulence and a Cascade of Airfoils with Non-Uniform Mean Flow," Journal of Fluid Mechanics, Vol. 463, 2002, pp. 25-52.

${ }^{18}$ Kraichnan, R. H., "Diffusion by a Random Velocity Field," The Physics of Fluids, Vol. 13, No. 1, 1970, pp. 22-31.

${ }^{19}$ Bechara, W., Bailly, C., and Lafon, P., "Stochastic Approach to Noise Modelling for Free Turbulent Flows," AIAA Journal, Vol. 32, No. 3, 1994, pp. 244-463.

${ }^{20}$ Bailly, C. and Juve, D., "A Stochastic Approach to Compute Subsonic Noise Using Linearized Euler's Equations," 5th AIAA/CEAS Aeroacoustics Conference, Greater Seatle, Washington, No. AIAA 99-1872, 1999.

${ }^{21}$ Billson, M., Eriksson, L.-E., and Davidson, L., "Jet Noise Modeling Using Synthetic Anisotropic Turbulence," 10th AIAA/CEAS Aeroacoustics Conference, Manchester, United Kingdom, No. AIAA 2004-3028, 2004.

${ }^{22}$ Reboul, G. and Polacsek, C., "Toward Numerical Simulation of Fan Broadband Noise Aft Radiation from Aeroengines," AIAA Journal, Vol. 48, No. 9, 2010, pp. 2038-2048.

${ }^{23}$ Klein, M., Sadiki, A., and Janicka, J., "A Digital Filter Based Generation of Inflow Data for Spatially Developing Direct Numerical or Large Eddy Simulations," Journal of Computational Physics, Vol. 186, 2003, pp. 652-665.

${ }^{24}$ Ewert, R. and Emunds, R., "CAA Slat Noise Studies Applying Stochastic Sound Sources Based On Solenoidal Digital Filters," 11th AIAA/CEAS Aeroacoustics Conference, Monterey, California, 2005.

${ }^{25}$ Dieste, M., Random-Vortex-Particle Methods Applied to Broadband Fan Interaction Noise, Ph.D. thesis, Institute of Sound and Vibrational Research, University of Southampton, 2011.

${ }^{26}$ Zhang, X., Chen, X. X., and Nelson, P. A., "Computation of Spinning Modal Radiation from an Unflanged Duct," AIAA Journal, Vol. 42, No. 6, 2004, pp. 1795-1801.

${ }^{27}$ Cheong, C., Joseph, P., and Lee, S., "High Frequency Formulation for the Acoustic Power Spectrum Due to CascadeTurbulence Interaction," Journal of the Acoustic Society of America, Vol. 119, No. 1, 2006, pp. 108-122.

${ }^{28}$ Paterson, R. W. and Amiet, R. K., "Noise and Surface Pressure Response of an Airfoil to Incident Turbulence," $J$. Aircraft, Vol. 14, No. 8, 1977, pp. 729-736.

${ }^{29}$ Amiet, R. K., "Noise Due to Turbulent Flow Past a Trailing Edge," Journal of Sound and Vibration, Vol. 47, 1976, pp. 387-393. 